Delayed onset of non-ischemic cerebral enhancing lesions is a rare complication of intracranial aneurysms' endovascular therapy (EVT). This complication has been attributed either to foreign body emboli and subsequent granulomatous reaction or cerebral hypersensitivity and nickel allergy. After retrospective review of all patients managed by EVT at our Institution from January 1st 2012 to December 31st 2014, 2 out of 374 patients $(0.5 \%)$ with such a complication were identified. Patient \# 1 developed non-ischemic cerebral enhancing (NICE) lesions 1 month after balloon assisted coiling of a ruptured anterior communicating artery aneurysm. Patient \# 2 developed NICE lesions 12 months (the longest delay reported to date for such complication) after the treatment of a right carotidophthalmic aneurysm by loose coiling and flow diversion. Skin patch testing was performed with all endovascular devices used in the 2 patients and with the European baseline series, including nickel. Patient \# 2 demonstrated nickel skin reactivity but none of the 2 patients presented allergic reaction to the devices used during interventions. Based on our observations and review of the literature, we hypothesize that delayed non-ischemic cerebral enhancing lesions after EVT are more likely related to foreign body emboli rather than nickel allergy.

Disclosures E. Shotar: None. B. Law-Ye: None. F. Di Maria: None. F. Baronnet-Chauvet: None. S. Zeidan: None. D. Psimaras: None. F. Bielle: None. C. Pecquet: None. S. Navarro: None. C. Rosso: None. F. Cohen: None. J. Chiras: None. N. Sourour: 2; C; Covidien. F. Clarençon: 2; C; Covidien, Codman.

\section{P-021 VERY DELAYED MONOCULAR BLINDNESS FOLLOWING FLOW DIVERSION TREATMENT OF OPHTHALMIC ARTERY ANEURYSM}

${ }^{1} \mathbf{T}$ Higashimori, ${ }^{1} \mathrm{D}$ Sandhu, ${ }^{1} \mathrm{f}$ Kim, ${ }^{2} \mathrm{~A}$ Grande, ${ }^{3} \mathrm{M}$ Ezzeddine, ${ }^{2} \mathrm{R}$ Tummala, ${ }^{4} \mathrm{~B}$ Jagadeesan. 'Neurology, Neurosurgery and Radiology, University of Minnesota, Minneapolis, MN; ${ }^{2}$ Neurosurgery, University of Minnesota, Minneapolis, MN; ${ }^{3}$ Neurology, University of Minnesota, Minneapolis, MN; ${ }^{4}$ Radiology, University of Minnesota, Minneapolis, MN

\subsection{6/neurintsurg-2016-012589.63}

Introduction Flow diversion is widely used for the effective endovascular treatment of wide-necked intracranial internal carotid artery (ICA) aneurysms. Ophthalmic artery (OA) occlusion is a known consequence related to flow diversion; however, OA occlusion in this setting usually is without clinical sequela. All reported cases of monocular vision loss after flow diversion have occurred within days to weeks of treatment.

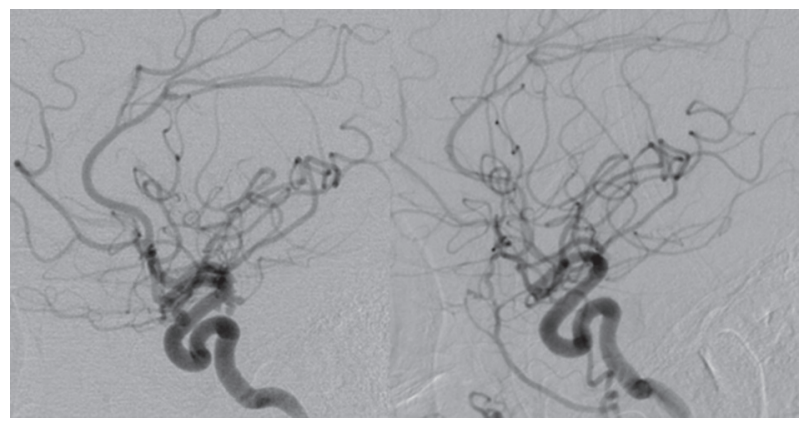

Abstract P-021 Figure 1
Methods We describe a case of monocular blindness due to ophthalmic artery occlusion nearly 3 years after flow diversion treatment of tandem supraclinoid ICA aneurysms. The medical records and images were reviewed for this patient.

Results This 51 year-old woman with history of hypertension was found to have two incidental right supraclinoid ICA aneurysms following headache workup. Cerebral angiography revealed a wide neck $5 \mathrm{~mm}$ saccular ophthalmic aneurysm and $3 \mathrm{~mm}$ bi-lobed posterior communicating artery (PCoA) aneurysm. Treatment with flow diversion was performed. A 4 $\times 20 \mathrm{~mm}$ Pipeline Embolization Device (PED) was deployed from the ICA terminus to the distal genu of the cavernous segment ICA without complication. She had been on dual antiplatelet therapy for 3 months followed by aspirin monotherapy. Six-month follow up angiography revealed obliteration of PCoA aneurysm, but with persistent filling of the ophthalmic aneurysm. Twenty months later, angiography again revealed persistent filling of the ophthalmic aneurysm. Both studies revealed patent right ophthalmic artery.

She presented with acute onset of complete right vision loss 32 months after PED placement. MRI brain showed no acute stroke. Dilated eye exam revealed cherry red spot in macula with attenuated vessels, and retinal whitening, consistent with ophthalmic artery occlusion. Diagnostic cerebral angiography revealed occlusion of the right ophthalmic artery with patent ICA and well apposed PED without in-stent stenosis or thrombosis. The ophthalmic aneurysm was no longer filling. There were no middle meningeal collaterals to the ophthalmic artery. IA tPA was given locally near the ophthalmic artery origin with minimal improvement. She was compliant with her aspirin throughout.

Conclusion Monocular vision loss remains a risk, even months to years following flow diversion of ICA aneurysms. Long term clinical follow-up is necessary to define the incidence of this complication.

Disclosures T. Higashimori: None. D. Sandhu: None. J. Kim: None. A. Grande: None. M. Ezzeddine: None. R. Tummala: None. B. Jagadeesan: None.

\section{P-022 THE ROAD LESS TRAVELED: TRANSARTERIAL EMBOLIZATION OF DURAL ARTERIOVENOUS FISTULAS VIA THE ASCENDING PHARYNGEAL ARTERY}

B Gross, F Albuquerque, K Moon, C McDougall. Neurosurgery, Barrow Neurological Institute, Phoenix, AZ

\subsection{6/neurintsurg-2016-012589.64}

Introduction With the introduction of Onyx, transarterial embolization has become the most common endovascular approach for dural arteriovenous fistulas (dAVF)s, often via the middle meningeal or occipital arteries. The ascending pharyngeal artery (APA) is a less frequently explored transarterial route as a result of its small caliber, potential anastomoses to the internal carotid and vertebral arteries, and its vital supply to lower cranial nerves.

Methods We reviewed our endovascular database (January 1996 - March 2016) for cranial dAVFs, evaluating dAVF characteristics and embolization results for those treated transarterially via the APA.

Results Of 267 endovascularly-treated dAVFs, 68 had APA supply (25\%). This included all marginal sinus dAVFs (11/11), $43 \%$ of transverse/sigmoid (37/86), 26\% of tentorial/petrosal 